\title{
IDENTIFICAÇÃO DO DIAGNÓSTICO DE ENFERMAGEM "RISCO DE QUEDAS EM IDOSOS COM ACIDENTE VASCULAR CEREBRAL”
}

\author{
Huana Carolina Cândido MORAIS ${ }^{a}$, Gabrielle Fávaro HOLANDA ${ }^{\mathrm{b}}$, Ana Railka de Souza OLIVEIRAc,
} Alice Gabrielle de Sousa COSTA ${ }^{\mathrm{d}}$, Camila Monique Bezerra XIMENES ${ }^{\mathrm{e}}$, Thelma Leite de ARAUJO ${ }^{\mathrm{f}}$

\section{RESUMO}

Estudo proposto para verificar a presença do diagnóstico de enfermagem (DE) "Risco de quedas de idosos com acidente vascular cerebral (AVC)". Trata-se de estudo observacional, exploratório, transversal, com análise descritiva, realizado em uma associação beneficente de reabilitação em Fortaleza/CE, Brasil, de janeiro a março de 2010, por fonte primária, com entrevista e exame físico, com idosos que tiveram pelo menos um episódio de AVC. Participaram 37 indivíduos: 20 (54,1\%) mulheres, média de 70,6 anos; 18 (48,6\%) residiam com companheiro, média de 5,2 anos de estudo. O Risco de quedas foi identificado em todos os idosos. Dentre os fatores de risco identificados, destacaram-se: Equilíbrio prejudicado (100\%), Idade acima de 65 anos (83,7\%) e Déficit proprioceptivo (83,7\%). Os enfermeiros devem considerar o risco de quedas como um dos enfoques do cuidado de enfermagem, bem como implementar e avaliar os resultados de intervenções relativas à prevenção de quedas.

Descritores: Diagnóstico de enfermagem. Fatores de risco. Acidentes por quedas. Acidente cerebral vascular. Idoso.

\section{RESUMEN}

El propósito del estudio fue evaluar la presencia del diagnóstico de enfermería riesgo de caídas en pacientes ancianos con accidente cerebrovascular. Observacional, exploratorio, descriptivo y transversal. Desarrollado en asociación de rehabilitación en Fortaleza, de enero hasta marzo del 2010 por entrevistas de fuentes primarias y examen fisico, hecho en personas con por lo menos un episodio de accidente cerebrovascular. 37 personas participaron, de los cuales 20 (54,1\%) eran mujeres, con 70,6 años como promedio, 18 (48,6\%) vivían con compañero, promedio de 5,2 años de estudio. El riesgo de caídas se encuentra en todas las personas mayores. Entre los factores de riesgo identificados se resalta: alteración del equilibrio (100\%), Mayores de 65 años (83,7\%) y Déficit propioceptivo (83,7\%). Las enfermeras deben considerar el riesgo de caídas como el foco de atención de enfermería, así como desarrollar y evaluar los resultados de intervenciones para la prevención de caídas.

Descriptores: Diagnóstico de enfermería. Factores de riesgo. Accidentes por caída. Accidente cerebrovascular. Anciano. Título: Identificación del diagnóstico de enfermería riesgo de caídas en ancianos con accidente cerebrovascular.

\section{ABSTRACT}

The purpose of this study was to verify the presence of a nursing diagnosis of fall risk in elderly with stroke. Observational, exploratory and cross-sectional study with descriptive analysis. Performed in a charitable rehabilitation association in Fortaleza city, from January to March 2010, by primary source, by interviews and physical examination with elderly people who had at least one episode of stroke. 37 individuals participated, of which 20 (54.1\%) were women, with mean age of 70.6 years, and 18 (48.6\%) lived with a partner and had an average of 5.2 years of study. The risk of falls was found in all elderly. Among the risk factors identified, it is possible to highlight: Impaired balance (100\%), Age above 65 years (83.7\%), and Proprioceptive deficit (83.7\%). Nurses must consider the risk of falls as a nursing care focus and implement and evaluate the results of interventions for fall prevention.

Descriptors: Nursing diagnosis. Risk factors. Accidental falls. Stroke. Aged.

Title: Identification of the nursing diagnosis of fall risk in elderly with stroke.

a Enfermeira. Aluna do curso de Mestrado em Enfermagem do Programa de Pós-Graduação em Enfermagem da UFC. Bolsista da Fundação Cearense de Apoio ao Desenvolvimento Científico e Tecnológico (FUNCAP). Vinculada ao Projeto Cuidado em Saúde Cardiovascular/ Conselho Nacional de Desenvolvimento Científico e Tecnoçógico (CNPq). Fortaleza, Ceará, Brasil.

b Enfermeira. Aluna do curso de Mestrado em Enfermagem do Programa de Pós-Graduação em Enfermagem da UFC. Bolsista da Coordenação de Aperfeiçoamento de Pessoal de Nível Superior (CAPES). Vinculada ao Projeto Cuidado em Saúde Cardiovascular/CNPq. Fortaleza, Ceará, Brasil.

c Enfermeira. Mestre em Enfermagem. Aluna do curso de Doutorado em Enfermagem do Programa de Pós-Graduação em Enfermagem da UFC. Bolsista CAPES. Vinculada ao Projeto Cuidado em Saúde Cardiovascular/CNPq. Fortaleza, Ceará, Brasil.

d Enfermeira. Mestre em Enfermagem. Aluna do curso de Doutorado em Enfermagem do Programa de Pós-Graduação em Enfermagem da

UFC. Bolsista FUNCAP. Vinculada ao Projeto Cuidado em Saúde Cardiovascular/CNPq. Fortaleza, Ceará, Brasil.

e Enfermeira assistencial da Clínica Pronefron. Vinculado ao Projeto Cuidado em Saúde Cardiovascular/CNPq. Fortaleza, Ceará, Brasil.

f Doutora em Enfermagem. Professora Associada do Departamento de Enfermagem da UFC. Fortaleza, Ceará, Brasil. 


\section{INTRODUÇÃO}

O envelhecimento acelerado da população brasileira aumenta a necessidade de cuidados em saúde de idosos, principalmente para aqueles em situações especiais, por exemplo, acometidos por acidente vascular cerebral (AVC), cujas sequelas acrescidas de alterações crônico-degenerativas próprias da idade, contribuem para o aumento de quedas ${ }^{(1)}$.

São condições comuns nesses indivíduos a presença de desequilíbrio postural, alterações sensoriais, motoras e dificuldade de locomoção. Dentre as consequências desses problemas, destacam-se as quedas, que representam a primeira causa de acidentes em pessoas com mais de 60 anos de idade ${ }^{(2)}$. No Brasil, anualmente calcula-se que $29 \%$ dos idosos sofrem quedas, sendo que $13 \%$ apresentam mais de um episódio por ano $^{(3)}$.

Idosos com AVC são mais suscetíveis à ocorrência de quedas, pois, muitas vezes, apresentam hemiplegia ou paresia dos membros inferiores, os quais afetam a marcha do indivíduo e sua capacidade de equilíbrio, além do aparecimento de disfunção visual, com prejuízo da deambulação segura ${ }^{(2)}$.

Os dados sobre a frequência de AVC não são exatos: calcula-se que, entre $14 \%$ e $39 \%$ dos acometidos tiveram uma ou mais quedas durante o período de internação hospitalar. Entre estes idosos, cerca de três quartos sofreram quedas nos seis meses subsequentes à alta hospitalar ${ }^{(4)}$. Um estudo identificou $16 \%$ de quedas de idosos no período de reabilitação após AVC, sendo que 1,2\% resultou em fraturas ${ }^{(5)}$.

Entretanto, apesar de ser fonte de morbidade e mortalidade de idosos, especialmente após um AVC, a queda pode ser evitada. Assim, a inferência do diagnóstico de enfermagem (DE) Risco de quedas, proposto pela NANDA-Internacional (NANDA $-\mathrm{I})^{(6)}$, representa um importante auxílio para evitar esses episódios em idosos, pois é uma das etapas da sistematização da assistência de enfermagem e orienta e define o planejamento das intervenções.

O DE defini-se como suscetibilidade aumentada a quedas que podem causar dano físico. É composto por definição e fatores de risco agrupados nas categorias: ambientais, cognitivos, fisiológicos, medicamentosos e específicos de adultos ${ }^{(6)}$.

Em relação à enfermagem, identificada a clientela sujeita a risco de quedas, o enfermeiro deve implementar ações visando à diminuição, ou mesmo supressão de ocorrência do evento. Para isso, são necessárias mais pesquisas para adaptação de instrumentos específicos para indivíduos após AVC. Instrumentos com essa finalidade não foram encontrados pelos pesquisadores do estudo. Outro aspecto relevante é um maior incentivo e treinamento do uso desses instrumentos por enfermeiros assistenciais em sua rotina diária.

Diante disso, a proposta do estudo foi identificar o diagnóstico de enfermagem Risco de quedas em idosos após AVC, presente na NANDA-I ${ }^{(6)}$.

\section{MÉTODO}

O estudo caracterizou-se como descritivo e transversal, e foi desenvolvido em uma unidade de reabilitação, com indivíduos após AVC, de janeiro a março de 2010, em Fortaleza, CE, Brasil. Referida instituição tem caráter filantrópico e atende ao nível de atenção ambulatorial, desenvolvendo atividades de atenção básica e de média complexidade.

Recrutaram-se os participantes mediante técnica de amostragem não-probabilística por conveniência. Foram incluídos pacientes com diagnóstico médico confirmado de AVC e idade igual ou acima de 60 anos. Excluíram-se os incapazes de ficar em pé, mesmo com auxílio, pela impossibilidade de realização do teste de equilíbrio e marcha; assim como pessoas com déficit cognitivo impossibilitadas de responder a formulário. A amostra corresponde à população, pois todos os indivíduos que compareceram na unidade no período da coleta de dados, e atenderam aos critérios de inclusão e exclusão, foram arrolados para o estudo.

Em consonância com os aspectos éticos de pesquisa científica com seres humanos, expressos na Resolução 196/96, do Ministério da Saúde( ${ }^{(7)}$, o estudo foi aprovado pelo Comitê de Ética em Pesquisa da Universidade Federal do Ceará (protocolo ${ }^{\circ}$ 314/09). Os participantes foram informados sobre os objetivos estabelecidos e assinaram o termo de consentimento livre e esclarecido.

Coletaram-se as informações por fonte primária, com entrevista e exame físico. $\mathrm{Na}$ entrevista, obtiveram-se dados sociodemográficos (sexo, idade, escolaridade, renda, situação conjugal, quantidade de pessoas por domicílio), informações clínicas (presença de fatores de risco que não puderam ser mensurados, como doença vascular, diabetes, falta de sono, entre outros) e fatores ambientais (pouca iluminação, quarto não familiar, ausência de material antiderrapante no banheiro, etc) relacionados com a ocorrência de quedas que compunham o DE Risco de quedas. 
$\mathrm{O}$ exame físico teve como finalidade avaliar os fatores físicos relacionados com a ocorrência de quedas: força muscular dos membros inferiores, equilíbrio e marcha, déficit proprioceptivo, capacidade visual e auditiva, déficit cognitivo e condições dos pés. Essa estratégia diminuiu a subjetividade de alguns fatores de risco, pois permitiu a avaliação dos pesquisadores com a utilização de instrumentos validados.

Assim, na parte do exame físico que avaliou a força muscular dos membros inferiores, observou-se a capacidade do paciente de flexionar ou estender os membros contra resistência ${ }^{(8)}$. Observaram-se movimentos de flexão e extensão de joelho e coxa; dorsiflexão, flexão plantar, inversão e eversão de tornozelos e pés.

Utilizou-se a "Escala de avaliação de marcha e equilíbrio de Tinetti”, em que se atingem 17 pontos de equilíbrio e 11 para marcha. Com relação ao escore obtido nos dois aspectos considerados da escala, quando inferior a 19 pontos, indica risco cinco vezes maior de quedas ${ }^{(1)}$. Tal ponto de corte classificou o indivíduo quanto à mobilidade física prejudicada.

Por não se encontrar escala validada e de fácil utilização para classificação do déficit proprioceptivo, convencionou dizer-se presente se o participante fosse portador de alguma paralisia, como hemiplegia ou hemiparesia. Para avaliar as condições dos pés utilizaram-se os métodos de inspeção e palpação para identificar a presença de calosidade, unha encravada, dedos em garra ou edema.

Utilizou-se a escala optométrica de Snellen para avaliar dificuldades visuais, pela facilidade de aplicação e compreensão dos alfabetizados e não-alfabetizados. Cada olho foi examinado separadamente, permitindo-se o uso de lentes corretivas. Os valores abaixo de 0,3 , em pelo menos um dos olhos, representam dificuldade visual ${ }^{(9)}$.

A capacidade auditiva obteve-se pelo Teste do Sussurro, no qual o examinador cobre o ouvido não testado, e sussurra palavras à distância de 30 a $60 \mathrm{~cm}$ do ouvido não-ocluído e fora da visão do paciente que repete corretamente o sussurrado ${ }^{(8)}$.

$\mathrm{O}$ déficit cognitivo foi avaliado com o Mini Exame do Estado Mental (MEEM), escore de 0 a 30 pontos e influenciado pelo nível de escolaridade. Adotaram-se como pontos de corte: 19 para analfabetos, 23 para um a três anos de estudo, 24 para quatro a sete anos de escolaridade e 28 para mais de sete anos de estudo ${ }^{(10)}$.
As informações serviram para inferência diagnóstica realizada pelos pesquisadores do estudo, considerando que a identificação de um fator de risco, indica a presença de DE Risco de quedas presente na Taxonomia II da NANDA-I. Essa classificação multiaxial reúne diagnósticos de enfermagem reais ou potenciais referentes a pessoas, famílias ou comunidades. Um DE potencial é composto por definição e fatores de risco.

Os dados foram compilados no software Excel e a análise realizada mediante abordagem estatística descritiva, para a qual se fez distribuição de frequências absolutas e relativas para variáveis categóricas, e de médias com desvio padrão (DP) para variáveis contínuas. Para melhor visualização, os dados foram apresentados em tabelas.

\section{RESULTADOS}

Participaram do estudo 37 indivíduos, 54,1\% do sexo feminino, com média de 70,6 anos $( \pm 6,37)$. Indivíduos com idade acima de 65 anos representaram $83,7 \%$. A média de estudos foi de $5,2( \pm 3,6)$ anos, com renda média mensal em torno de 708,37 reais $( \pm 500,37)$, todos aposentados, $48,6 \%$ residiam com companheiro e média de 3,72 pessoas $( \pm 1,8)$ por domicílio; $5,4 \%$ moravam sozinhos.

O DE Risco de quedas foi identificado em $100 \%$ dos participantes, uma vez que todos possuíam pelo menos um fator de risco para o evento.

A Tabela 1 apresenta a distribuição dos participantes segundo fatores de risco ambientais para o DE Risco de quedas.

A maioria dos participantes relatou residir em locais com reduzidos fatores de risco ambientais de queda, com poucos móveis, adequadamente iluminados, e material antiderrapante no piso do banheiro.

A Tabela 2 dispõe as condições de desempenho cognitivo dos participantes segundo o MEEM.

Os participantes foram distribuídos conforme anos de estudo: $43,3 \%$ estudaram de 4 a 7 anos; enquanto $27 \%$, de 1 a 3 anos. Considerando-se exclusivamente o maior grupo (4 a 7 anos de estudo), $55 \%$ apresentaram déficit cognitivo. Enquanto, no segundo maior grupo ( 1 a 3 anos de estudo), $25 \%$ tinham déficit cognitivo. Em síntese, $54 \%$ dos pacientes avaliados apresentaram déficit cognitivo, com escore médio de 23,64 pontos $( \pm 3,39)$.

História de quedas nos últimos seis meses foi relatada por $40,5 \%$ dos avaliados e $54 \%$ afirmaram não utilizar dispositivos auxiliares de locomoção. 
Tabela 1 - Distribuição dos participantes segundo fatores de risco ambientais no domicílio. Fortaleza, 2010

\begin{tabular}{lcc}
\hline Variáveis & N & \% \\
\hline \multicolumn{4}{l}{ Ambiente com móveis e objetos } & em & excesso \\
\hline Sim & 14 & $37,8 \%$ \\
Não & 23 & $62,2 \%$ \\
Total & 37 & $100,0 \%$
\end{tabular}

Ausência de material antiderrapante no piso do banheiro

$\begin{array}{lll}\text { Sim } & 18 & 48,6 \% \\ \text { Não } & 19 & 51,4 \% \\ \text { Total } & 37 & 100,0 \%\end{array}$

Ausência de material antiderrapante no chão do box do chuveiro

$\begin{array}{lll}\text { Sim } & 18 & 48,6 \% \\ \text { Não } & 19 & 51,4 \% \\ \text { Total } & 37 & 100,0 \%\end{array}$

Pouca iluminação

\begin{tabular}{lll} 
Sim & 14 & $37,8 \%$ \\
Não & 23 & $62,2 \%$ \\
Total & 37 & $100,0 \%$ \\
Quarto não familiar & & \\
Sim & 1 & $2,7 \%$ \\
Não & 36 & $97,3 \%$ \\
Total & 37 & $100,0 \%$ \\
\hline
\end{tabular}

Na tabela 3 representam-se os fatores de risco fisiológicos, para o referido diagnóstico.

Déficit proprioceptivo foi o fator de risco fisiológico mais presente (83,7\%); 13,5\% possuíam alteração auditiva, e 51,4\% apresentaram dificuldade visual. Identificaram-se alterações nos pés em $35,2 \%$ dos participantes, sendo observada a presença de calosidade, unha encravada, dedos em garra e edema. Como Outros foram agrupados fatores de risco fisiológico menos presentes na amostra: doença vascular $(10,8 \%)$, vertigem ao virar/estender o pescoço $(8,1 \%)$, anemias, condições pós-operatórias e diarreia $(5,4 \%)$ e doença aguda $(2,7 \%)$.

$\mathrm{Na}$ Tabela 4 apresentam-se os resultados da Escala de avaliação de marcha e equilíbrio de Tinetti.

O escore final médio foi de 19,4 pontos $( \pm 6,78)$. Dos participantes $43,3 \%$ apresentaram mobilidade física prejudicada. Os avaliados tinham equilíbrio prejudicado, assim, ninguém atingiu a pontuação máxima da escala. Enquanto 78,4\% apresentaram dificuldade para marcha, apenas $21,6 \%$ mantiveram integridade da força muscular.

Como medicamentos, que constituem fatores de risco para quedas, foi mais citado o uso de agentes anti-hipertensivos $(51,4 \%)$, diuréticos e inibidores da enzima conversora da angiotensina $(46 \%)$. Antidepressivos tricíclicos e hipnóticos foram menos relatados, $8,1 \%$ e $2,7 \%$, respectivamente.

\section{DISCUSSÃO}

$\mathrm{O}$ risco de quedas esteve presente em todos os idosos, independente de sexo, motivo pelo qual sempre deve ser investigado, uma vez que esse grupo etário apresenta mais predisposição para incapacidade funcional e doenças crônicas. As mulheres foram maioria devido à elevada esperança de vida, e também por formarem uma população maior do que a de homens no Brasil, razões porque são mais acometidas pelos efeitos negativos do envelhecimento, entre eles, as quedas ${ }^{(11)}$.

Em relação à influência dos fatores socioeconômicos sobre o risco de quedas, ainda não existe um consenso. Estudo realizado com idosos investigou fatores associados a quedas em três grupos, compostos por aqueles que não caíram no decorrer do ano, aqueles que caíram uma única vez e os que apresentaram quedas recorrentes; como resultado não foram encontradas diferenças significantes quanto aos dados sociodemográficos ${ }^{(12)}$. Outra pesquisa mostrou que quedas em idosos foram estatisticamente significantes no sexo feminino, nos mais velhos, separados, divorciados e viúvos e nos de nível socioeconômico mais baixo $(\mathrm{p}=0,04)^{(13)}$.

São causadores de quedas fatores intrínsecos ao próprio indivíduo e extrínsecos, relacionados ao ambiente. Os fatores de risco ambientais podem se modificar de forma eficaz, caso o enfermeiro assuma sua função de promover um ambiente seguro ${ }^{(3)}$, modificando-os, especialmente, por meio de estratégias de visita domiciliar e de educação em saúde.

Os problemas com o ambiente são tão mais perigosos quanto maior a vulnerabilidade do idoso e sua instabilidade. Na maioria dos eventos, os idosos não caem por realizar atividades perigosas, mas no desenvolvimento de atividades rotineiras ${ }^{(3)}$. Identificar fatores de risco ambientais presentes no DE Risco de quedas possibilita uma intervenção do enfermeiro no sentido de eliminar esses fatores e evitar eventos de quedas. 
Tabela 2 - Resultados do Mini-Exame do Estado Mental conforme o grau de escolaridade. Fortaleza, 2010

\begin{tabular}{|c|c|c|c|c|c|c|c|c|}
\hline \multirow{3}{*}{$\begin{array}{l}\text { Anos de } \\
\text { estudo }\end{array}$} & \multicolumn{2}{|c|}{ Total } & \multicolumn{2}{|c|}{ Escores do MEEM } & \multicolumn{4}{|c|}{ Classificação } \\
\hline & \multirow[t]{2}{*}{$\mathbf{N}$} & \multirow[t]{2}{*}{$\%$} & \multirow[t]{2}{*}{ Intervalo } & \multirow[t]{2}{*}{ Média } & \multicolumn{2}{|c|}{ Normal } & \multicolumn{2}{|c|}{ Déficit Cognitivo } \\
\hline & & & & & $\mathbf{N}$ & $\%$ & $\mathbf{N}$ & $\%$ \\
\hline $\mathrm{O}$ & 3 & $8,1 \%$ & $23-27$ & 25,3 & 3 & $17,6 \%$ & $\mathrm{O}$ & $\mathrm{O} \%$ \\
\hline $1-3$ & 10 & $27 \%$ & $17-26$ & 22,1 & 5 & $29,4 \%$ & 5 & $25 \%$ \\
\hline $4-7$ & 16 & $43,3 \%$ & $18-28$ & 22,8 & 5 & $29,4 \%$ & 11 & $55 \%$ \\
\hline$>7$ & 8 & $21,6 \%$ & $21-30$ & 26,6 & 4 & $23,6 \%$ & 4 & $20 \%$ \\
\hline Total & 37 & $100 \%$ & $17-30$ & 23,64 & 17 & $100 \%$ & 20 & $100 \%$ \\
\hline
\end{tabular}

Uma locomoção eficaz é possível quando o sistema perceptivo está capacitado a extrair do ambiente informação relevante para estabilização e realização de ações corretivas, em meio ao desequilíbrio corporal ${ }^{(9)}$. Isso é possível utilizando-se visão, audição e músculos. Objetos e móveis em excesso, associados à iluminação insuficiente do ambiente, aumentam o número de quedas por dificultarem a interpretação do espaço pelo indivíduo.

Fisiologicamente, os idosos apresentam diminuição na capacidade para enxergar imagens com pouco brilho e cor, prejudicando o contraste e a nitidez visual. A deficiência repercute no controle postural, pois as informações da visão ajudam a interpretar o ambiente de forma mais eficaz, permitindo uma locomoção segura ${ }^{(14)}$.

Estudo observou que as dificuldades visuais apresentadas pela população configuram-se como importante fator de risco para a ocorrência de quedas, especialmente em idosos, e quando associados a outros fatores tem seu efeito potencializado ${ }^{(15)}$. Confirma-se a correlação entre o número de quedas de idosos e os valores da Tabela de Snellen, justificada pelo fato de idosos com baixa visão terem idade mais avançada e pior mobilidade em relação aos com visão normal ${ }^{(14)}$.

Quedas de pacientes após AVC ocorrem, especialmente, no ambiente domiciliar, 59\% no quarto, enquanto, $18 \%$ no banheiro ${ }^{(5)}$. Assim, utilizar material antiderrapante no piso do banheiro é imprescindível para diminuição dos eventos. Neste estudo, 48,6\% dos participantes não utilizam esse material, estando mais suscetíveis a quedas no banheiro. Entretanto é importante salientar a natureza subjetiva dos achados, por serem originados somente de informações do entrevistado e não de avaliação do ambiente pelos pesquisadores.
Tabela 3 - Distribuição dos participantes segundo fatores de risco fisiológicos. Fortaleza, 2010

\begin{tabular}{lll}
\hline Variáveis & $\mathbf{N}$ & \% \\
\hline Artrite & & \\
Sim & 8 & $21,6 \%$ \\
Não & 29 & $78,4 \%$ \\
Total & 37 & $100,0 \%$
\end{tabular}

\section{Déficit Proprioceptivo}

$83,7 \%$

Não

$16,3 \%$

Total

$100 \%$

Doença vascular

Sim

Não

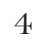

$10,8 \%$

Total

$89,2 \%$

$100,0 \%$

\section{Falta de Sono}

Sim

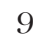

$24,3 \%$

Não

$75,7 \%$

Total

Hipotensão ortostática
Sim

Não

Total

Incontinência urinária

Sim

Não

Total

Diabetes

Sim

Não

Total
$27 \%$

$73 \%$

$100,0 \%$
$32,4 \%$

$67,6 \%$

$100,0 \%$
$21,6 \%$

$78,4 \%$

$100,0 \%$ 
Continuação.

\begin{tabular}{lll}
\hline Variáveis & $\mathbf{N}$ & $\mathbf{\%}$ \\
\hline Dificuldades auditivas & & \\
Sim & 5 & $13,5 \%$ \\
Não & 32 & $86,5 \%$ \\
Total & 37 & $100,0 \%$ \\
Dificuldades visuais & & \\
Sim & 19 & $51,4 \%$ \\
Não & 18 & $48,6 \%$ \\
Total & 37 & $100,0 \%$ \\
Outros & & \\
Sim & 10 & $27 \%$ \\
Não & 27 & $73 \%$ \\
Total & 37 & $100,0 \%$ \\
\hline
\end{tabular}

O risco de queda é agravado pelo estado mental rebaixado, entendido como limitação da função cognitiva, o qual representa um dos maiores problemas dos idosos. Esses indivíduos que apresentam possíveis comprometimentos físicos e cognitivos do AVC podem ter esse estado agravado por uma queda, aumentando sua dependência pela perda da autonomia e diminuição de suas capacidades funcionais, resultando em sobrecarga de familiares e cuidadores como consequência das quedas ${ }^{(15)}$.

Apresentaram déficit cognitivo 54\% dos participantes, de acordo com o MEEM. Estudo realizado com idosos acerca da recorrência de quedas encontrou diferença significante nos escores do MEEM, entre o grupo sem quedas e o grupo com quedas recorrentes. Observou-se que o declínio cognitivo é forte fator de risco para quedas, pois o idoso apresenta dificuldade para executar atividades, sofre com desorientação espacial e suas respostas protetoras estão comprometidas, assim como prejuízo na marcha, equilíbrio e estabilidade postural ${ }^{(12)}$.

Entre os fatores de risco fisiológicos investigados, destacam-se incontinência urinária (32,4\%), déficit proprioceptivo (83,7\%), dificuldades visuais $(51,4 \%)$, e diminuição da força nas extremidades inferiores (78,4\%). A incontinência urinária, comum na faixa etária, provoca problemas psicológicos e sociais, com perda da autoestima e isolamento social. Sua prevalência na população maior de 65 anos de idade é de 15 a 30\%. Pesquisa demonstra alta prevalência entre idosos, principalmente mulheres, e correlação inversa com a perda de mobilidade e função cognitiva prejudicada. Não foi constatada associação com a idade ${ }^{(16)}$.

Propriocepção é a capacidade dada ao sistema nervoso central de receber e processar informações sensoriais de receptores presentes nas estruturas responsáveis pela movimentação e orientação. As informações discriminam a posição e o movimento articular, inclusive a direção, amplitude, velocidade e a tensão relativa sobre os tendões. Obtém-se como resultado a consciência da posição e movimentação, estabilização e proteção da articulação, mediante sinais reflexos mediados pela medula espinhal e manutenção do equilíbrio e postura, atuando como fator protetor contra quedas ${ }^{(17)}$.

Fraqueza de membros inferiores é fator de risco em destaque. O problema causa diminuição de mobilidade funcional, para realização das atividades de vida diária, predispondo a maior chance de quedas ${ }^{(15)}$.

A redução da força muscular nas extremidades inferiores afeta a capacidade para realizar atividades motoras e adaptação ao ambiente, contribuindo para ocorrência de instabilidades e quedas. É relevante orientar a população acerca da importância de realizar atividades físicas para diminuição da fraqueza de membros inferiores ${ }^{(2,11)}$.

Todos os entrevistados apresentaram algum grau de alteração no equilíbrio e/ou marcha. Corroborando a informação, pesquisa anterior mostra que $55 \%$ de quedas relacionam-se com alterações de marcha; $32 \%$, com alterações de equilíbrio e demais com fatores extrínsecos, como superfícies irregulares, escadas e sapatos inapropriados ${ }^{(18)}$.

Marcha é o movimento rítmico que mantém o corpo em locomoção progressiva. Resulta do perfeito equilíbrio entre forças externas que agem no corpo e de forças internas dos músculos, tendões, ossos, ligamentos e articulações ${ }^{(18)}$. A manutenção do equilíbrio e do controle postural são necessários para o desempenho adequado das atividades habituais. A deterioração do equilíbrio com a idade é bem definida pela literatura e idosos com déficit de equilíbrio são mais propensos a quedas e suas consequências $^{(14)}$.

Diante das várias morbidades observadas nos idosos, especialmente naqueles após AVC, é grande a quantidade de medicamentos utilizados, o que pode aumentar a ocorrência de efeitos adversos ou interações medicamentosas. O número de medicamentos utilizados é forte preditor para quedas, 
Tabela 4-Classificação dos indivíduos mediante Escala de avaliação de marcha e equilíbrio Tinetti. Fortaleza, 2010

\begin{tabular}{lll}
\hline Variáveis & N & \% \\
\hline Escore de Tinetti & & \\
$<19$ & 16 & $43,3 \%$ \\
$\geq 19$ & 21 & $56,7 \%$ \\
Total & 37 & $100,0 \%$ \\
Equilíbrio prejudicado & & \\
Sim & 37 & $100 \%$ \\
Não & 0 & $0 \%$ \\
Total & 37 & $100,0 \%$ \\
Dificuldade na Marcha & & \\
Sim & 29 & $78,4 \%$ \\
Não & 8 & $21,6 \%$ \\
Total & 37 & $100,0 \%$ \\
\hline
\end{tabular}

portanto é necessário maior atenção quanto à prescrição de uso e ajuste de dosagem ${ }^{(12)}$. Destaca-se o uso de anti-hipertensivos, citado por $51,4 \%$ dos participantes, uma vez que tais medicamentos são medidas de prevenção do AVC e dificilmente podem ser dispensados, corroborando o risco de quedas ${ }^{(19)}$.

As classes medicamentosas mais utilizadas e que aumentam o risco de quedas, são as de diuréticos, inibidores da enzima conversora de angiotensina (ECA), beta-bloqueadores e bloqueadores dos canais de cálcio. Como reações adversas citam-se: câimbras, sensação de fraqueza muscular, tonturas e hipotensão ortostática, levando a alterações nos mecanismos de marcha e equilíbrio ${ }^{(19)}$.

Outra classe investigada foi a dos psicofármacos. Revisão sistemática mostrou que dos psicofármacos os que mais se relacionam com quedas são os benzodiazepínicos, neurolépticos e antidepres$\operatorname{sivos}^{(20)}$. No presente estudo, poucos participantes utilizavam esses medicamentos.

É fundamental que o enfermeiro tenha conhecimento farmacológico para avaliar os efeitos das medicações utilizadas pelos pacientes, e orientar sobre sua administração, interações e contraindicações, além de efeitos adversos ${ }^{(19)}$. As medidas instrumentalizam o idoso para evitar ou diminuir os fatores de risco de quedas, de forma mais consciente.

Em face disso, a prevenção de quedas é de responsabilidade dos cuidadores, familiares e pro- fissionais de saúde. É possível diminuir o risco de quedas com os seguintes cuidados na promoção da saúde: revisão dos medicamentos; modificações nos domicílios; promoção da segurança no domicílio; promoção da segurança fora do domicílio ${ }^{(13)}$.

\section{CONCLUSÕES}

O DE Risco de quedas foi identificado em todos os idosos avaliados, com destaque para os fatores de risco: equilíbrio prejudicado (100\%), idade acima de 65 anos $(83,7 \%)$, déficit proprioceptivo $(83,7 \%)$, força diminuída nas extremidades inferiores $(78,4 \%)$, dificuldades na marcha $(78,4 \%)$, estado mental rebaixado (54\%), dificuldades visuais $(51,4 \%)$ e uso de anti-hipertensivos $(51,4 \%)$. Os achados confirmam a origem multifatorial e complexa do evento queda em idosos após AVC. O enfermeiro, na prática profissional, deve reconhecer os fatores de risco, e ser capaz de minimizar seus efeitos, adotando intervenções adequadas e avaliando os resultados obtidos na prevenção de quedas.

Apesar das evidências o estudo tem limitação, por haver sido realizado com um grupo com características clínicas específicas de participantes em único momento. Recomenda-se sua replicação em grupos maiores, com caráter longitudinal, possibilitando investigação de outras variáveis e correlação com a ocorrência de quedas.

\section{REFERÊNCIAS}

1 Tinetti ME. Performance-oriented assessment of mobility problems in elderly patients. J Am Geriatr Soc. 1986;34(2):119-126.

2 Menezes RL, Bachion MM. Estudo da presença de fatores de riscos intrínsecos para quedas, em idosos institucionalizados. Cienc Saude Coletiva. 2008;13(4):1209-1218.

3 Nascimento FA, Vareschi AP, Alfieri FM. Prevalência de quedas, fatores associados e mobilidade funcional em idosos institucionalizados. Arq Catarin Med. 2008;37(2):7-12.

4. Ashburn A, Hyndman D, Pickering R, Yardley L, Harris S. Predicting people with stroke at risk of falls. Age Ageing. 2008;37:270-276.

5 Lee JE, Stokic DS. Risk factors for falls during inpatient rehabilitation. Am J Phys Med Rehabil. 2008;87:341-353. 
6 North American Nursing Diagnosis Association (NANDA). Diagnósticos de enfermagem da NANDA: definições e classificação 2009-2011. Porto Alegre: Artmed; 2010.

7 Conselho Nacional de Saúde (Br). Resolução n ${ }^{\circ}$ 196/96. Decreto no 93.333 de janeiro de 1987. Estabelece critérios sobre pesquisa envolvendo seres humanos. Bioética. 1996;4(supl. 2):15-25.

8 Smeltzer SC, Bare BG, organizadores. Brunner \& Suddarth - tratado de enfermagem medico-cirúrgica. 10 a ed. Rio de Janeiro: Guanabara Koogan; 2005.

9 Luiz LC, Rebelatto JR, Coimbra AMV, Ricci NA. Association between visual deficit and clinical-functional characteristics among community-dwelling older adults. Rev Bras Fisioter. 2009; $13(5): 444-450$.

10 Ministério da Saúde (BR), Secretaria de Atenção à Saúde. Departamento de Atenção Básica. Envelhecimento e saúde da pessoa idosa. Brasília (DF), 2006.

11 Navarro FM, Rabelo JF, Faria ST, Lopes MCL, Marcon SS. Percepção de idosos sobre a prática e a importância da atividade física em suas vidas. Rev Gaúcha Enferm. 2008; 29(4):596-603.

12 Ricci NA, Gonçalves DFF, Coimbra IB, Coimbra AMV. Fatores associados ao histórico de quedas de idosos assistidos pelo programa de saúde da família. Saúde Soc São Paulo. 2010;19(4):898-909.

13 Siqueira FV, Facchini LA, Piccini RX, Tomasi E, Thumé E, Silveira DS, et al. Prevalência de quedas em idosos e fatores associados. Rev Saude Publica. 2007;41(5):749-756.

14. Macedo BG, Pereira LSM, Gomes PF, Silva JP, Castro ANV. Impacto das alterações visuais nas quedas, desempenho funcional, controle postural e no equilíbrio dos idosos: uma revisão de literatura. Rev Bras Geriatr Gerontol. 2008;11(3):419-432.

15 Costa AGS, Oliveira ARS, Moreira RP, Cavalcante TF, Araujo TL. Identificação do risco de quedas em idosos após acidente vascular encefálico. Esc Anna Nery. 2010;14(4):684-689.

16 Busato Jr WFS, Mendes FM. Incontinência urinária entre idosos institucionalizados: relação com mobilidade e função cognitiva. Arq Catarin Med. 2007;36(4):49-55.

17 Martimbianco ALC, Polachini LO, Chamlian TR, Masiero D. Efeitos da propriocepção no processo de reabilitação das fraturas de quadril. Acta Ortop Bras. 2008;16(2):112-116.

18 Kirkwood RN, Araújo PA, Dias CS. Biomecânica da marcha em idosos caidores e não caidores: uma revisão da literatura. Rev Bras Cienc Mov. 2006;14(4):103-110.

19 Silva LD, Henrique DM, Schutz V. Ações do enfermeiro na terapia farmacológica para o acidente vascular cerebral: uma revisão integrativa. Rev Enferm UERJ. 2009; $17(3): 423-429$.

20 Gama ZAS, Goméz-Conesa A. Factores de riesgo de caídas em ancianos: revisión sistemática. Rev Saúde Pública. 2008;42(5):946-956.

\author{
Endereço do autor / Dirección del autor / \\ Author's address: \\ Huana Carolina Cândido Morais \\ Rua Cento e dois, 74, Conjunto Esperança \\ 60763-510, Fortaleza, CE \\ E-mail: huanacarolina@yahoo.com.br \\ Telefone: 5585 3296-2012 | 8855-0589.
}

